\title{
FAMILIES OF SOLUTIONS OF THE UNILATERAL MATRIX EQUATION
}

JAMES H. BELL

1. Introduction and summary. Let $\mathcal{F}$ be a field of characteristic zero, and let $R_{m}(m=0,1, \cdots, r)$ be $n \times n$ matrices with elements in F. M. H. Ingraham [1 $]^{1}$ has shown that the unilateral matrix equation $\sum_{m=0}^{r} R_{m} X^{m}=0$ may have an infinitude of solutions. In this paper a study is made of the conditions under which such an infinitude of solutions will exist. All matrices considered in this paper will be square and of order $n$ unless otherwise specified.

The matrix $X_{1}$ is similar to the matrix $X_{2}$ if there exists a nonsingular matrix $T$ such that $T X_{1} T^{-1}=X_{2}$ and the elements of $X_{1}, X_{2}$ and $T$ are in $\mathcal{F}$. The polynomial domain $\mathcal{F}[\lambda]$ of $\mathcal{F}$ is a principal ideal ring. The matrix $\sum_{m=0}^{r} R_{m} \lambda^{m}$ is a $\lambda$-matrix, that is, its elements are in $\mathcal{F}[\lambda]$. A square $\lambda$-matrix is unimodular if it has an inverse which is also a $\lambda$-matrix. Two $\lambda$-matrices $A$ and $B$ are left associates if there is a unimodular matrix $U$ such that $U A=B$. The relationship of left associativity is an equals relationship. Every square $\lambda$-matrix is a left associate of a unique matrix which is in canonical triangular form. This matrix has all elements below the main diagonal equal to zero. If a diagonal element is zero, its row consists entirely of zeros. The leading coefficient of each nonzero diagonal element is unity, and the elements in that column are reduced modulo the diagonal element. ${ }^{2}$

If $M, P$, and $A$ are $\lambda$-matrices such that $M=P A$, then $A$ is said to be a right divisor of $M$, and $M$ is a left multiple of $A$. Every pair of $\lambda$-matrices has a greatest common right divisor (g.c.r.d.) $D$ and every pair of nonsingular $\lambda$-matrices has a least common left multiple (l.c.l.m.) $M$ which is unique up to left associates [2].

The question arises: Given two nonsingular matrices $A$ and $B$, what other right divisors of their l.c.l.m. $M$ are there which are not left associates of $A$ or $B$ ? It has been shown ${ }^{2}$ that $X_{1}$ is a solution 1949.

Presented to the Society, September 7, 1948; received by the editors January 21,

1 Numbers in brackets refer to the bibliography at the end of the paper.

${ }^{2}$ For these definitions and terms see MacDuffee [2]. The Hermite normal form as defined by MacDuffee is the form generally used. The canonical triangular form defined here is used in keeping with that used by Ingraham [1]. It is obtained in the same manner as the Hermite normal form except the operations are carried out in the columns in reverse order.

3 The proof of the factor theorem for polynomials with coefficients in a ring with an identity element is given by Albert [3]. 
of $\sum_{m=0}^{r} R_{m} X^{m}=0$ if and only if $\lambda I-X_{1}$ is a right divisor of $\sum_{m=0}^{r} R_{m} \lambda^{m}$. Therefore, if there exist two distinct solutions $X_{1}$ and $X_{2}$, the l.c.l.m. of $\lambda I-X_{1}$ and $\lambda I-X_{2}$ is a right divisor of $\sum_{m=0}^{r} R_{m} \lambda^{m}$. If this 1.c.l.m. has a right divisor of the form $\lambda I-X_{3}$, where $X_{3}$ is different from $X_{1}$ and $X_{2}$, then $X_{3}$ is another solution of the unilateral equation. Thus, a study of the above problem yields results applicable to the unilateral matrix equation. The $\lambda$-matrix $A$ is a right divisor of the $\lambda$-matrix $M$ if and only if its canonical triangular form is a right divisor of the canonical triangular form of $M$. Consequently, the matrices $A, B$, and $M$ will be considered as being in canonical triangular form.

If the $\lambda$-matrix $R(\lambda)$ has a set of right divisors whose canonical triangular forms have the same main diagonal, then the set will be called a family of right divisors of $R(\lambda)$. A matrix of the form $\lambda I-X$ where $X$ has elements in $\mathcal{F}$ will be called a monic matrix of degree one. It is easily shown that two monic matrices are left associates if and only if they are identical. If a family of right divisors of $R(\lambda)$ are the left associates of monic matrices of degree one, the matrices $X$ obtained from the monic matrices are solutions of $R(X)=0$ and will be called a family of solutions of $R(X)=0$.

It is shown that a necessary and sufficient condition for the existence of an infinite family of solutions of the unilateral matrix equation is that there be two distinct solutions which are similar. All matrices similar to a given solution are solutions of the equation if and only if their minimal polynomial is a common divisor of the elements of $\sum_{m=0}^{r} R_{m} \lambda^{m}$. An interesting result arises as a by-product of this second result, namely: The equation $\sum_{m=0}^{r} R_{m} \cdot \times X^{m}=0$ ( $\sum_{m=0}^{r} X^{m} \cdot \times R_{m}=0$ ), where $R_{m} \cdot \times X^{m}$ is the direct product of $R_{m}$ and $X^{m}[2]$, has a solution if and only if the elements of $\sum_{m=0}^{r} R_{m} \lambda^{m}$ have a greatest common divisor which is not a unit. (In this problem the order of $X$ is not restricted to $n$.) It is also shown that if $\sum_{m=0}^{r} R_{m} X^{m}=0$ has a solution, and if the determinant of $\sum_{m=0}^{r} R_{m} \lambda^{m}$ is zero identically, then there is an infinitude of solutions of the equation.

2. Families of solutions. The following theorem provides a basis for the results obtained in this paper.

Theorem 1. If $P=Q A$, where $P$ is a nonsingular $\lambda$-matrix, $P$ and $A$ being in canonical triangular form, and if for some values $j$ and $k$, where $j>k$, it is true that $q_{k k}$ and $a_{j j}$ are not relatively prime; then there exists a family of right divisors of $P$ which are not left associates. Furthermore, if $A$ is the left associate of a monic matrix of degree one, then the mem- 
bers of the family, with a finite number of exceptions, are also left associates of monic matrices of degree one.

Since $P$ and $A$ are triangular matrices and $P$ is nonsingular, it follows that $Q$ and $A$ are nonsingular, and $Q$ is also triangular. Hence, $p_{m m}=q_{m m} a_{m m}(m=1,2, \cdots, n)$.

Consider a possible factorization of $P$ into $P=B A^{\prime}$, where $A^{\prime}$ has the same diagonal elements as $A$ and is in canonical triangular form. One such factorization exists, namely $P=Q A$. The matrix $B$ will be a triangular matrix.

Represent the greatest common divisor of two polynomials $f(\lambda)$ and $g(\lambda)$ by the symbol $(f(\lambda), g(\lambda))$.

Select the values of $k$ and $j$ so that: $\left(q_{k k}, a_{j j}\right) \neq 1$ and $j>k ;\left(q_{m m}, a_{p p}\right)$ $=1$ when $m<p$ and $p>j ;\left(q_{m m}, a_{j j}\right)=1$ for $m<k$. Also let $a_{m p}^{\prime}=a_{m p}$ $(m, p=1,2, \cdots, j-1 ; p=j, m=k+1, k+2, \cdots, j-1)$. Since $b_{m m}=q_{m m}(m=1,2, \cdots, n)$ and $b_{m p}=q_{m p}(m, p=1,2, \cdots, j-1$; $p=j, m=k+1, k+2, \cdots, j-1)$, the element $a_{k t}^{\prime}$ is obtained from the following equation:

$$
p_{k j}=q_{k k} a_{k j}^{\prime}+\sum_{m=k+1}^{+1} q_{k m} a_{m i}+b_{k j} a_{j j}
$$

that is,

$$
q_{k k} a_{k j}^{\prime} \equiv p_{k j}-\sum_{m=k+1}^{j-1} q_{k m} a_{m i} \bmod a_{j j}
$$

This congruence has a solution, namely $a_{k j}$. Therefore $\left(q_{k k}, a_{j j}\right)$ divides $p_{k j}-\sum_{m=k+1}^{j-1} q_{k m} a_{m j}$, and $a_{k j}^{\prime}=x_{1}(\lambda)+\left(\sum_{m=1}^{p} a_{m} \lambda^{p-m}\right) f_{1}(\lambda)$ where $x_{1}(\lambda)$ and $f_{1}(\lambda)$ are uniquely determined, $p$ is the degree of $\left(q_{k k}, a_{j j}\right)$ and $a_{m}(m=1,2, \cdots, p)$ are arbitrary parameters. The remaining elements of $A^{\prime}$ are uniquely determined in terms of $a_{k j}^{\prime}$ and the other elements previously found, since $\left(q_{m m}, a_{j j}\right)=1(m<k)$, and $\left(q_{m m}, a_{p p}\right)=1(p>j, p>m)$.

Therefore, in solving for $A^{\prime}$ an infinite family of right divisors of $P$ is obtained. Furthermore, each member of the family is in canonical triangular form. Hence, no two distinct members of the family may be left associates.

The existence of a family of right divisors of $P$ has been shown. In the construction above the parameters first occur in $a_{k j}^{\prime}$ and may occur in the elements $a_{m p}^{\prime}(m<k, p=j ; p=j+1, \cdots, n)$. When the parameters occur in an element, they will appear in the coefficients of the various powers of $\lambda$ in that element. In this case the coefficients will be polynomials in the parameters. A certain combination of values 
for the parameters will yield $A$ from $A^{\prime}$. Assign these values to all but one of the parameters. The modified matrix $A^{\prime}$ will involve only one parameter, and by assigning the proper value to this parameter $A$ will be obtained from $A^{\prime}$.

If there is a unimodular matrix $T$ such that $T A=\lambda I-X$, then it is true that $A^{\prime}$ will be the left associate of a matrix $\lambda I-X^{\prime}$ except possibly for a finite set of values of the parameter. The necessary and sufficient conditions that the canonical triangular matrix $A=\sum_{m=0}^{i} A_{m} \lambda^{m}$ be the left associate of a monic matrix of degree one are: The degree of $\prod_{m=1}^{n} a_{m m}$ should equal $n$, the degree of $\prod_{m=1}^{j} a_{m m}$ should be equal to or less than $j(j<n)$, and the matrix $W_{1}$ $=\left\|A_{8} A_{\imath-1} \cdots A_{1}\right\|$ shall be of rank $n$. Since $a_{m m}^{\prime}=a_{m m}(m=1,2, \cdots, n)$ the first conditions are satisfied. The matrix $W_{1}$ consists of at most $n$ nonzero columns. ${ }^{4}$ That is, the rank of $W_{1}$ is the rank of an $n \times n$ matrix $D$ formed from the nonzero columns of $W_{1}$. Because $|D|$ is a polynomial in the parameter and $|D| \not \equiv 0$ (since $T A=\lambda I-X$ ), it follows that $|D|=0$ for only a finite set of values of the parameter. Therefore there is an infinite family of right divisors of $\boldsymbol{P}$ which are left associates of monic matrices of degree one.

The following theorem is due to V. J. Varineau [4].

THEOREM 2. If the least common left multiple of two nonsingular matrices $A$ and $B$, with elements in a principal ideal ring, is $M$, and their greatest common right divisor is $D$; then $|M| \cdot|D|=u|A| \cdot|B|$ where $u$ is a unit.

TheOREM 3. The unilateral matrix equation $\sum_{m=0}^{r} R_{m} X^{m}=0$ has an infinite family of solutions if and only if there exist two distinct solutions $X_{1}$ and $X_{2}$ which are similar.

Let $A, B$, and $M$ be the canonical triangular forms of $\lambda I-X_{1}$, $\lambda I-X_{2}$, and the 1.c.l.m. of $\lambda I-X_{1}$ and $\lambda I-X_{2}$ respectively. Since $X_{1}$ and $X_{2}$ are solutions of $\sum_{m=0}^{r} R_{m} X^{m}=0, A$ and $B$ are right divisors of $\sum_{m=0}^{r} R_{m} \lambda^{m}$. Therefore $M$ is a right divisor of $\sum_{m=0}^{r} R_{m} \lambda^{m}$. It will be shown that $M$ has a family of right divisors of the form $\lambda I-X^{\prime}$, and thus $\sum_{m=0}^{r} R_{m} X^{m}=0$ has a family of solutions.

Since $|A|=|B|=\left|\lambda I-X_{1}\right|=\left|\lambda I-X_{2}\right|, M$ is nonsingular. Also $M=P A=Q B$, where $P$ and $Q$ are triangular $\lambda$-matrices. Either $a_{m m}=b_{m m}(m=1,2, \cdots, n)$ or else not all $a_{m m}=b_{m m}(m=1,2, \cdots, n)$. If $a_{m m}=b_{m m}(m=1,2, \cdots, n)$ it follows that $\left(p_{k k}, a_{j j}\right) \neq 1$ for some values $j$ and $k$ where $j>k$. If this were not so, there would be a

1 Published as note without proof in Ingraham [1]. Proof given in a paper by Bell [5]. 
unique right divisor of $M$ having the elements $a_{m m}$ as the main diagonal elements, whereas there are at least two such divisors, namely $A$ and $B$. The theorem follows from Theorem 1 .

Assume, in the second case, that not every $a_{m m}$ is equal to the corresponding $b_{m m}$. Let $j$ be the least value of $m$ such that $a_{j j} \neq b_{j j}$. Then $a_{j j}=f(\lambda) h(\lambda)$ and $b_{j j}=g(\lambda) h(\lambda)$ where $(f(\lambda), g(\lambda))=1$. Since $m_{j j}=p_{j j} a_{j j}=q_{j j} b_{j j}$ it follows that $g(\lambda)$ divides $p_{j j}$ and $f(\lambda)$ divides $q_{j j}$. Because $\prod_{m=-a_{m m}}^{n}=\prod_{m=1}^{n} b_{m m}$, it follows that some factor of $g(\lambda)$ divides some element $a_{p p}(p>j)$, and some factor of $f(\lambda)$ divides some element $b_{k k}(k>j)$. That is, $\left(b_{k k}, q_{j j}\right) \neq 1(k>j)$ and $\left(a_{p p}, p_{j j}\right) \neq 1(p>j)$. By Theorem 1, there exists a family of solutions whose corresponding canonical triangular forms have the same main diagonal as $A$, and another family, whose corresponding canonical triangular forms have the same main diagonal as $B$.

If there is a family of solutions, let $X_{1}$ be one of the solutions. All members of the family will satisfy the scalar equation $\left|\lambda I-X_{1}\right|=0$. A scalar equation of finite degree may have only a finite number of dissimilar solutions [2]. Therefore, there must exist at least two similar solutions. Furthermore, there must be at least one class of similar solutions which is infinite in number. This completes the proof of Theorem 3.

3. The solution of $\sum_{m=0}^{r} R_{m} \cdot X^{m}=0$. If $X_{1}$ is a solution of the unilateral equation and $X_{2}$ is a matrix similar to $X_{1}$, it does not follow that $X_{2}$ is a solution. Since $X_{2}=S X_{1} S^{-1}, X_{2}$ will be a solution if and only if $\sum_{m=0}^{r} R_{m} S X^{m}=0$. A nonzero, but not necessarily nonsingular, solution for $S$ will exist if and only if $\left|\sum_{m=0}^{r} R_{m} \cdot \times\left(X_{1}^{T}\right)^{m}\right|$ $=0$ (where $A \cdot \times B=\left(A b_{i j}\right)$ and $X^{T}$ is the transpose of $X$ ) [2]. The form of the matrix involved in this determinant creates an interesting problem namely: When does the equation $\sum_{m=0}^{r} R_{m} \cdot \times X^{m}=0$ have a solution? It should be noticed that $X$ need no longer be of order $n$.

THEOREM 4. The equation $\sum_{m=0}^{r} R_{m} \cdot \times X^{m}=0$, where $R_{m}$ $(m=0,1, \cdots, r)$ and $X$ have elements in $\mathcal{F}$, has a solution if and only if the elements of $\sum_{m=0}^{r} R_{m} \lambda^{m}$ have a greatest common divisor which is not a unit.

Consider the matrix $R_{m} \cdot \times X^{m}=\left(R_{m} x_{i j}^{(m)}\right)$ where $R_{m}=\left(r_{i j}^{(m)}\right)$ and $X^{m}=\left(x_{i}^{(m)}\right)$. By simply rearranging its elements this matrix may be changed into the matrix $\left(r_{v}^{(m)} X^{m}\right)$. If $\sum_{m=0}^{r} R_{m} \cdot \times X^{m}=0$, then $\sum_{m=0}^{r}\left(r_{w}^{(m)} X^{m}\right)=0$ and conversely. Therefore, $\sum_{m=0}^{r} r_{(m)}^{(m)} X^{m}=0$, and the solution $X$ must satisfy the scalar equation $\sum_{m=0^{r}}^{r}(m) X^{m}=0$, for each value of $i$ and $j$. Hence the elements of the matrix $\sum_{m=0}^{r} R_{m} \lambda^{m}$ 
must have the minimum polynomial of $X$ as a common divisor.

If the elements of $\sum_{m=0}^{r} R_{m} \lambda^{m}$ have a greatest common divisor which is not a unit, then the companion matrix of that polynomial will be a solution of the equations $\sum_{m=0^{-}(m)}^{r} X^{m}=0$ and hence will be a solution of $\sum_{m=0}^{r} R_{m} \cdot \times X^{m}=0$. Similar considerations show that the same condition is necessary and sufficient for the solution of $\sum_{m=0}^{r} X^{m}$ $\cdot \times R_{m}=0$. A solution of $\sum_{m=0}^{r} R_{m} \cdot \times X^{m}=0$ is a solution of $\sum_{m=0}^{r} X^{m} \cdot \times R_{m}=0$ and conversely.

COROLlary. The equations $\sum_{m=0}^{r} R_{m} \cdot \times X^{m}=0$ and $\sum_{m=0}^{r} X^{m} \cdot \times R_{m}$ $=0$ will have a solution of order $n$ if and only if the scalar equation formed by setting the g.c.d. of the elements of $\sum_{m=0}^{r} R_{m} \lambda$ equal to zero has $a$ solution of order $n$.

The above condition will certainly be true if the elements of $\sum_{m=0}^{r} R_{m} \lambda^{m}$ have a common divisor whose degree is a divisor of $n$.

THEOREM 5. If $X_{1}$ is a solution of the unilateral matrix equation $\sum_{m=0}^{r} R_{m} X^{m}=0$, then all matrices similar to $X_{1}$ are solutions if and only if the minimal polynomial of $X_{1}$ is a common divisor of the elements of $\sum_{m=0}^{r} R_{m} \lambda^{m}$.

If the minimal polynomial $g(\lambda)$ of $X_{1}$ is a common divisor of the elements of $\sum_{m=0}^{r} R_{m} \lambda^{m}$, it follows that $\sum_{m=0}^{r} R_{m} \lambda^{m}=Q(\lambda)[g(\lambda) I]$.

If $X_{2}$ is similar to $X_{1}$ then $g\left(X_{2}\right)=0$ or $g(\lambda) I=P(\lambda)\left(\lambda I-X_{2}\right)$. That is, $\sum_{m=0}^{r} R_{m} \lambda^{m}=Q(\lambda) P(\lambda)\left(\lambda I-X_{2}\right)$ and $X_{2}$ is a solution of the unilateral equation.

To prove the converse, let $X_{2}=S X_{1} S^{-1}$. Since every matrix similar to $X_{1}$ is a solution, it follows that $S$ is a solution of $\sum_{m=0}^{r} R_{m} S X_{1}^{m}=0$. This is true for every nonsingular $S$.

The equation $\sum_{m=0}^{r} R_{m} S X_{1}^{m}=0$ is equivalent to $n^{2}$ equations in the unknowns $s_{i j}(i, j=1,2, \cdots, n)$. The matrix $S$ may be considered as a vector in $n^{2}$ space. If there are $n^{2}$ nonsingular solutions $S$ of $\sum_{m=0}^{r} R_{m} S X_{1}^{m}=0$, and if these matrices, considered as vectors in $n^{2}$ space, are linearly independent, then it follows that $\sum_{m=0}^{r} R_{m}$ $\cdot \times\left(X_{1}^{T}\right)^{m} \equiv 0$.

Let $E_{i j}$ be the $n \times n$ matrix which has 1 in the $i$ th row and $j$ th column and zeros elsewhere. The $n^{2}-n$ matrices $I+E_{i j}(i \neq j ; i$, $j=1,2, \cdots, n)$ are linearly independent and nonsingular. The $n$ matrices $\sum_{m \neq i} E_{m m}+a_{i} E_{i i}(i=1,2, \cdots, n)$ are nonsingular and will be linearly independent if the $a_{i}(i=1,2, \cdots, n)$ are chosen so that the determinant of $S_{n}=\sum_{m=1}^{n} a_{m} E_{m m}+\sum_{m, p} E_{m p}(m \neq p ; m, p=1$, $2, \cdots, n)$ is not zero. This is obviously possible when $n=2$. A simple induction step suffices to show that it is possible for a general $n$. 
A total of $n^{2}$ linearly independent nonsingular matrices have been constructed above. If every $X_{2}$ similar to $X_{1}$ is a solution of $\sum_{m=0}^{r} R_{m} X^{m}=0$, then $\sum_{m=0}^{r} R_{m} S X_{1}^{m}=0$ has $n^{2}$ linearly independent solutions for $S$. Therefore, $\sum_{m=0}^{r} R_{m} \cdot \times\left(X_{1}^{T}\right)^{m} \equiv 0$, that is, $X_{1}^{T}$ is a solution of $\sum_{m=0}^{r} R_{m} \cdot \times X^{m}=0$. Theorem 5 follows from Theorem 4 since the minimal polynomial of $X_{1}^{T}$ is the minimal polynomial of $X_{1}$.

4. Infinite sets of solutions which are not families. There may be an infinite number of solutions of the unilateral equation without there being an infinite family. This fact is brought out in the proof of the following theorem.

Theorem 6. If $\left|\sum_{m=0}^{r} R_{m} \lambda^{m}\right| \equiv 0$, and if there exists a solution $X_{1}$ of the unilateral equation $\sum_{m=0}^{r} R_{m} X^{m}=0$, then there is an infinite number of solutions.

The canonical triangular matrix $P$ of $\sum_{m=0}^{r} R_{m} \lambda^{m}$ may be factored into the product $Q A$ where $A$ is the canonical triangular form of $\lambda I-X_{1}$, and $Q$ is triangular. Since $\left|\sum_{m=0}^{r} R_{m} \lambda^{m}\right|=\delta \prod_{m=1}^{n} p_{m m}=0$ ( $\delta$ a unit), it follows that some element $p_{i i}=0$, and therefore $q_{i i}=0$.

Factor $P$ into $Q^{\prime} A^{\prime}$ letting $a_{m m}^{\prime}=a_{m m}(m=1,2, \cdots, n)$, then $q_{m m}^{\prime}=q_{m m}$. Let $q_{k k}^{\prime}$ be the first diagonal element of $Q^{\prime}$ which is zero. Three possibilities arise in the factorization:

1. $\left(q_{y j}^{\prime}, a_{p p}^{\prime}\right) \neq 1$, for some values $p$ and $j$ such that $p>j$, and $q_{j j} \neq 0$.

2. Case 1 does not occur and $k<n$.

3. Case 1 does not occur and $k=n$.

In Case 1, as a result of Theorem 1, there exists an infinite family of solutions of the unilateral equation. The fact that some elements $q_{u}^{\prime}$ are zero may be taken care of by letting $a_{\imath_{\imath}, t+m}^{\prime}=a_{l, l+m}$.

If $q_{k k}^{\prime}=0$, that is $p_{k k}=0,(k<n)$, then $p_{k m}=0(m=k, k+1, \cdots, n)$. Since $a_{m m}^{\prime} \neq 0(m=1,2, \cdots, n)$ it follows that all $q_{k m}^{\prime}=0$. Choose $a_{l m}^{\prime}=a_{l m}(l, m=1,2, \cdots, n-1 ; l=n ; m=n, n-1, \cdots, k+1)$. The element $a_{k n}^{\prime}$ is obtained from the equation $\sum_{m=k}^{n} q_{k m}^{\prime} a_{m m}^{\prime}=0$. Since all $q_{k m}^{\prime}=0, a_{k n}^{\prime}$ may be chosen to be $a_{k n}$ with the coefficients of the powers of $\lambda$ replaced by parameters. Since $\left(q_{m m}^{\prime}, a_{n n}^{\prime}\right)=1(m<k)$, the remaining terms of $A^{\prime}$ may be expressed uniquely in terms of $a_{\mathbf{k}}^{\prime}$. As in Theorem 1 , it follows that there is a family of right divisors of $P$ with the same diagonal as $A$, and hence a family of solutions of the unilateral equation. In the final case $k=n$, and $\left(q_{m m}^{\prime}, a_{p p}\right)=1(m<p)$. Choose $a_{l m}^{\prime}=a_{l m}(l, m=1,2, \cdots, n-1)$. If $a_{n n}^{\prime}$ is chosen to be $a_{n n}$, a unique factorization is obtained namely $P=Q A$. Since $q_{n n}^{\prime}=0, a_{n n}^{\prime}$ may be chosen arbitrarily. Choose $a_{n n}^{\prime}$ to be identical with $a_{n n}$ except 
in the constant term which will be a parameter.

It may easily be shown that if there is given a finite set of irreducible polynomials, and a given polynomial of fixed degree with an arbitrary constant, then for an infinite set of values of the arbitrary constant, the polynomial will be relatively prime to all of the polynomials in the given finite set. This follows from an application of Euclid's algorithm and the fact that the field is of characteristic zero.

If the finite set of irreducible polynomials is taken to be the factors of the elements $q_{m m}^{\prime}(m=1,2, \cdots, n-1)$, then for an infinite set of values of the constant $\left(q_{m m}^{\prime}, a_{m m}^{\prime}\right)=1$. Since $\left(q_{m m}^{\prime}, a_{n n}\right)=1, a_{n n}$ will be one of the $a_{n n}^{\prime}$. A unique factorization of $P$ exists for each $a_{m \text {. }}^{\prime}$. Therefore, there is an infinite set of right divisors of $P$. Since $A$ is included in the set, there is an infinite set of right divisors of $P$ which are left associates of monic matrices of degree 1 . It follows that there is an infinite set of solutions of the unilateral equation.

Note that in Case 3 the set of solutions, as defined, is not a family.

It may be possible in certain cases to factor $P$ in such a way as to get a family of right divisors which leads to a family of solutions. That this is not always true is shown by the following example in which $\mathcal{F}$ is the rational field $R$. Let

$$
P=\left\|\begin{array}{ll}
\lambda^{2} & 1 \\
0 & 0
\end{array}\right\|
$$

There is no factorization of $P$ in $R[\lambda]$ which will yield an infinite family of right divisors, and solutions of the equation:

$$
\left\|\begin{array}{ll}
1 & 0 \\
0 & 0
\end{array}\right\| X^{2}+\left\|\begin{array}{ll}
0 & 1 \\
0 & 0
\end{array}\right\|=\left\|\begin{array}{ll}
0 & 0 \\
0 & 0
\end{array}\right\|
$$

However, since

$$
\left\|\begin{array}{cc}
\lambda^{2} & 1 \\
0 & 0
\end{array}\right\|=\left\|\begin{array}{cc}
\lambda & 1 / e \\
0 & 0
\end{array}\right\|\left\|\begin{array}{cc}
\lambda & -1 / e \\
0 & \lambda+e
\end{array}\right\|, \quad \quad e \neq 0,
$$

there is the infinite set of solutions:

$$
X=\left\|\begin{array}{cc}
0 & 1 / e \\
0 & -e
\end{array}\right\|
$$

\section{BIBLIOGRAPHY}

1. M. H. Ingraham, Rational methods in matrix equations, Bull. Amer. Math. Soc. vol. 47 (1941) pp. 61-70.

2. C. C. MacDuffee, The theory of matrices, Ergebnisse der Mathematik und ihrer Grenzgebiete, Chelsea, 1946, pp. 31-36, 81, 89, 94. 
3. A. A. Albert, Modern higher algebra, The University of Chicago Science Series, 1937 , p. 26.

4. V. J. Varineau, An extension of the theory of matrices with elements in a principal ideal ring, This paper was submitted as his doctoral dissertation at the University of Wisconsin, July 1940.

5. J. H. Bell, Left associates of monic matrices with an application to unilateral matrix equations, Amer. J. Math. vol. 71 (1949) pp. 249-256, see p. 254.

Michigan State College

\section{CANONICAL POSITIVE DEFINITE MATRICES UNDER INTERNAL LINEAR TRANSFORMATIONS}

\section{BERNARD VINOGRADE}

Let $P$ be a real positive definite $n$-rowed square matrix, $n=p+q$ $+r, p \leqq q \leqq r, S=\operatorname{diag}\left(S_{1}, S_{2}, S_{3}\right)$, where $S_{1}$ is $p$-rowed, $S_{2}$ is $q$-rowed, and $S_{3}$ is $r$-rowed, all nonsingular. $S$ is called an internal linear transformation when it transforms $P$ according to the rule $P \rightarrow S P S^{\prime}$. All coefficients are real numbers. The problem is to find a canonical form for $P$, under internal linear transformations, depending on characteristic value systems associated with $P$. The partition of $n$ into three parts is for convenience only.

1. Semi-canonical reduction. Partition $P$ according to the partition of $n$, and call the square diagonal blocks $P_{1}, P_{2}$, and $P_{3}$, with $p, q$, and $r$ rows respectively. Choose $S_{1}=Q_{1}^{-1}, S_{2}=Q_{2}^{-1}, S_{3}=Q_{3}^{-1}$ from the factorizations $P_{1}=Q_{1} Q_{1}^{\prime}, P_{2}=Q_{2} Q_{2}^{\prime}, P_{3}=Q_{3} Q_{3}^{\prime}$. Then

$$
S P S^{\prime}=Q=\left(\begin{array}{ccc}
I_{p} & A & B \\
A^{\prime} & I_{q} & C \\
B^{\prime} & C^{\prime} & I_{r}
\end{array}\right),
$$

where $I_{i}$ is the $i$-rowed identity matrix. Any further transformations will be chosen so as to preserve the diagonal blocks $I_{p}, I_{q}, I_{r}$. Hence we shall have $S_{i} S_{i}^{\prime}=I_{i}, i=1,2,3$.

2. Non-special cases. Let $X=\left(I_{p}, A, B\right), \quad Y=\left(A^{\prime}, I_{q}, C\right), Z$ $=\left(B^{\prime}, C^{\prime}, I_{r}\right)$, so $X X^{\prime}, Y Y^{\prime}$, and $Z Z^{\prime}$ are all nonsingular and symmetric. Let their characteristic roots be the ordered diagonal elements of $D_{1}=\operatorname{diag}\left(\lambda_{1}, \cdots, \lambda_{p}\right), D_{2}=\operatorname{diag}\left(\mu_{1}, \cdots, \mu_{q}\right)$, and $D_{3}$

Received by the editors December 27, 1948. 